University of South Carolina

Scholar Commons

$3-2001$

\title{
Bistable Operation of a Two-Section 1.3-mm InAs Quantum Dot Laser-Absorption Saturation and the Quantum Confined Stark Effect
}

Xiaodong Huang

A. Stintz

Hua Li

Audra Rice

G. T. Liu

See next page for additional authors

Follow this and additional works at: https://scholarcommons.sc.edu/emec_facpub

Part of the Mechanical Engineering Commons, Nanoscience and Nanotechnology Commons, Optics Commons, and the Quantum Physics Commons

Publication Info

Postprint version. Published in IEEE Journal of Quantum Electronics, Volume 37, Issue 3, 2001, pages 414-417.

(C) IEEE Journal of Quantum Electronics, 2001, IEEE

Huang, X., Stintz, A., Li, H., Rice, A., Liu, G.T., Lester, L.F., Cheng, J., Malloy, K.J. (2001). Bistable Operation of a Two-Section 1.3-mm InAs Quantum Dot Laser-Absorption Saturation and the Quantum Confined Stark Effect. IEEE Journal of Quantum Electronics, 37(3), 414-417.

This Article is brought to you by the Mechanical Engineering, Department of at Scholar Commons. It has been accepted for inclusion in Faculty Publications by an authorized administrator of Scholar Commons. For more information, please contact digres@mailbox.sc.edu. 


\section{Author(s)}

Xiaodong Huang, A. Stintz, Hua Li, Audra Rice, G. T. Liu, L.F. Lester, Julian Cheng, and K.J. Malloy 


\title{
Bistable Operation of a Two-Section 1.3- $\mu \mathrm{m}$ InAs Quantum Dot Laser-Absorption Saturation and the Quantum Confined Stark Effect
}

\author{
Xiaodong Huang, Member, IEEE, A. Stintz, Hua Li, Audra Rice, G. T. Liu, Student Member, IEEE, \\ L. F. Lester, Member, IEEE, Julian Cheng, Senior Member, IEEE, and K. J. Malloy, Member, IEEE
}

\begin{abstract}
Room temperature, continuous-wave bistability was observed in oxide-confined, two-section, 1.3- $\mu \mathrm{m}$ quantum-dot (QD) lasers with an integrated intracavity quantum-dot saturable absorber. The origin of the hysteresis and bistability were shown to be due to the nonlinear saturation of the QD absorption and the electroabsorption induced by the quantum confined Stark effect.
\end{abstract}

Index Terms-Absorption saturation, electroabsorption, hysteresis, optical bistability, quantum confined Stark effect, quantum-dot lasers, quantum dots, saturable absorber, semiconductor laser.

\section{INTRODUCTION}

$\mathbf{S}$ ELF-ASSEMBLED InAs or InGaAs quantum dots (QDs) have been used as the gain medium to achieve laser diodes on GaAs substrates, with very low threshold current densities and emission wavelengths up to $1.3-\mu \mathrm{m} \mathrm{[1]-[5].}$ The properties of the QD layers as an absorber in an active device have not been investigated in detail, however, and only weak (bias circuit dependent) bistability and self-pulsation have been reported in a shorter wavelength QD laser with an intracavity QD saturable absorber [6]. Optical bistability is usually associated with two-section laser structures where one section is actively pumped while the other acts as a saturable absorber. This bistable behavior has important applications in optical switching and modulation [7], [8]. More importantly, 1.3- $\mu \mathrm{m}$ QDs have been shown to exhibit ultrafast dynamic response [9], which suggests an ultrafast recovery time for a QD saturable absorber. An ultrafast QD saturable absorber may lead to high speed optical switching and modulation and to new techniques for semiconductor mode-locking. In this paper, we report the bistable operation of a long wavelength oxide-confined two-section QD laser with an integrated intracavity absorber formed with the same QD layers, in which both absorption saturation and electroabsorption induced by the quantum confined Stark effect (QCSE) play a role.

\section{DeVice StRUCTURE AND FABRICATION}

The devices were fabricated from the same wafer as described in [5]. The wafer was grown by solid-source MBE on

Manuscript received August 30, 2000.

The authors are with the Center for High Technology Materials, University of New Mexico, Albuquerque, NM 87106 USA (e-mail: xdhuang@chtm.unm.edu).

Publisher Item Identifier S 0018-9197(01)01631-1.

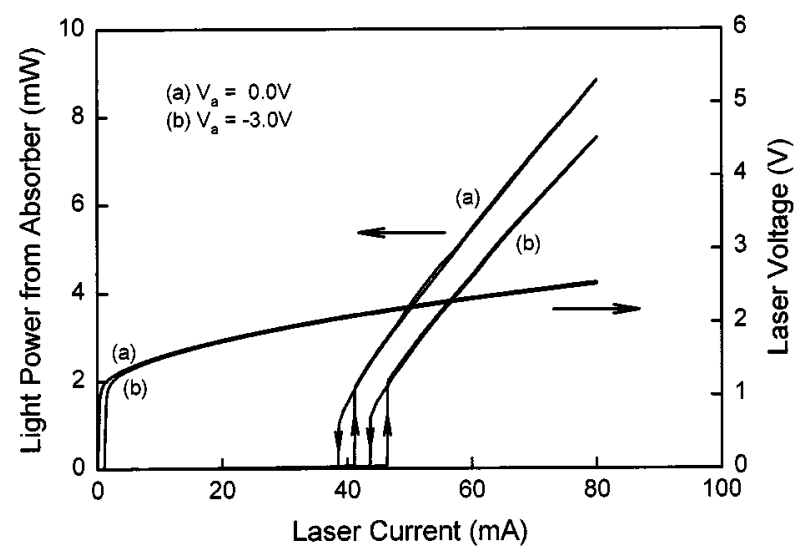

Fig. 1. Room-temperature $\mathrm{CW}$ lasing and electrical characteristics of a two-section QD laser with: (a) $V_{a}=-0.0 \mathrm{~V}$ short circuit and (b) a reverse bias of $V_{a}=-3.0 \mathrm{~V}$, applied to the absorber section.

a (001) $n^{+}$-GaAs substrate. The active region consists of two InAs quantum-dots-in-a-well (DWELL) layers [2] separated by a $30 \mathrm{~nm}$ GaAs barrier layer, situated in the middle of a 220-nm-thick GaAs waveguide bounded by $\mathrm{Al}_{0.7} \mathrm{Ga}_{0.3} \mathrm{As}$ cladding layers. The devices have a typical two-section laser structure with a $50-\mu \mathrm{m}$ gap in the top $p$-type contact metals. The lengths of the gain section and the absorber section are $L_{g}=4.73$ and $L_{a}=1.79 \mathrm{~mm}$, respectively, which ensure ground state lasing. An isolation resistance of $2.86 \mathrm{k} \Omega$ is achieved between these two sections by using shallow dry etching to remove the heavily doped cap layer in the gap region. Current confinement is provided by the wet lateral oxidation [10] of a 50 -nm-thick $\mathrm{Al}_{0.98} \mathrm{Ga}_{0.02}$ As layer positioned between the waveguide and the upper cladding layers, resulting in a narrow current aperture of $10 \mu \mathrm{m}$. Detailed device processing has been reported in [5]. No coating is applied to the cleaved facets. The devices were mounted on a copper heat sink with the $p$-side up, and were tested under continuous-wave (CW) lasing operation at room temperature.

\section{EXPERIMENTAL RESULTS}

The devices were measured with current injection $\left(I_{g}\right)$ into the gain section and a constant reverse bias voltage $\left(V_{a}\right)$ applied to the absorber section. Room-temperature CW lasing occurred on the QD ground state. The peak external efficiency exceeded $25 \%$ for one facet and the lasing wavelength measured just above threshold is $1285 \mathrm{~nm}$. Fig. 1 shows how the lasing 
light power $(L)$ emitted from the absorber facet and the voltage $\left(V_{g}\right)$ across the laser section vary with the forward and backward sweep of the laser current under: 1) short-circuit conditions, with $V_{a}=0.0 \mathrm{~V}$ and 2) reverse bias, with $V_{a}=-3.0$ $\mathrm{V}$ across the absorber section. The $L-I_{g}$ characteristics exhibit clear counterclockwise hysteresis loops and bistability. The loop position shifts to a higher laser current with increased reverse bias, while the loop width increases. The on-off ratio is about 27:1 at the center of the hysteresis loop. Unlike the devices described in [6], the appearance of bistability in this device does not require a resistive load in the absorber bias circuit to provide feedback via the photocurrent change. Only a constant reverse bias voltage was applied to the absorber section. When the gain section is biased in the middle of the hysteresis loop, on-off switching and modulation can be attained by applying a positive or a negative current pulse to the gain section. Note that the $V_{g}-I_{g}$ characteristic for $V_{a}=-3.0 \mathrm{~V}$ is displaced due to the leakage current between the gain section and the absorber section.

From the threshold conditions at the corners of the hysteresis loop, the modal loss of the absorber was estimated as a function of the light power out of the absorber under different reverse bias conditions, as shown in Fig. 2. The internal loss and the laser gain versus current density values obtained in [5] were used in the calculation with the leakage current subtracted from the threshold current. As expected, the modal loss of the absorber is comparable to the modal gain of the laser section. The absorption inside the absorber decreases nonlinearly as the light power output of the absorber is increased. Since the average light intensity within the absorber is proportional to the lasing light power output of the absorber, Fig. 2 also shows that the absorption of light by the absorber decreases nonlinearly as the light intensity inside the absorber is increased. This nonlinear saturation of absorption with increasing light intensity originates from the emptying of the QD ground state transition [11], [12], and is the origin of the hysteresis and bistability in the light power versus current characteristics. The saturation of absorption with increased light output introduces positive feedback and causes the sudden rise and fall of light power during the forward and backward current sweep. At higher injection current levels, gain saturation and the large difference between the unsaturated and saturated loss of the absorber under higher reverse bias result in a wider hysteresis loop. Finally, Fig. 2 also indicates the presence of electroabsorption, as absorption increases with increasing reverse bias on the absorber.

Hysteresis and bistability were also observed upon applying a constant laser injection current and varying the reverse bias voltage on the absorber. Fig. 3 shows how the light power out of the absorber and the photocurrent induced in the absorber vary with forward and backward sweep of the reverse bias voltage on the absorber. A clear and wide hysteresis loop exists in the light power versus reverse bias voltage characteristic, which is the combined result of both absorption saturation and the electroabsorption effect. This hysteresis and bistability appear not to have been reported in two-section quantum well (QW) lasers, although absorption saturation and electroabsorption can also simultaneously occur in a QW [13]. The total absorber current has two components-a leakage current between the gain sec-

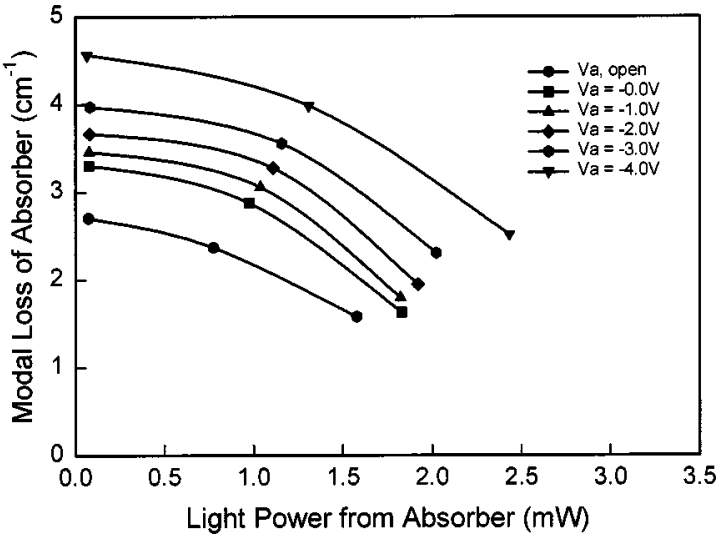

Fig. 2. Calculated modal loss inside the absorber as a function of light power out of the absorber under open circuit conditions $\left(V_{a}=1.165 \mathrm{~V}\right)$ and under a reverse bias of $V_{a}=0.0,-1.0,-2.0,-3.0,-4.0 \mathrm{~V}$, respectively, across the absorber.

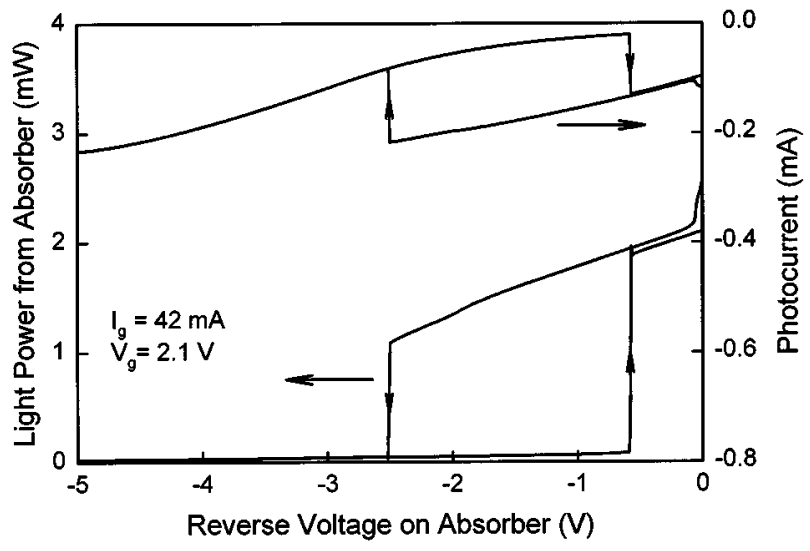

Fig. 3. Light power output of the absorber and its photocurrent under the forward and backward sweeps of the reverse bias voltage, showing hysteresis loops. The laser drive current is kept constant at $I_{g}=42 \mathrm{~mA}$.

tion and the absorber section, and the photocurrent due to electron-hole pair creation via the absorption of light. The photocurrent in Fig. 3 was obtained by subtracting the leakage current from the total absorber current. Hysteresis loops also exist in the photocurrent characteristics corresponding to the loop in the light power, and there is a very large negative differential resistance corresponding to the sudden changes in light power. A device with the absorber biased in the middle of the hysteresis loop can be switched on and off by using a positive or negative voltage pulse, respectively. The reverse-biased absorber requires no current injection, the photocurrent change is quite small, and QD dynamics are intrinsically fast [9]. Therefore, using a shorter absorber section with better electrical isolation between the gain section and the absorber, high-speed switching and modulation controlled only by the absorber reverse bias are possible [14].

To explore the electroabsorption effect further, the absorption spectra were measured using the technique described in [15]. First, as shown in Fig. 4(a), the electroluminescence spectra out of the absorber was measured under different reverse bias conditions, while the laser is biased approximately at transparency 


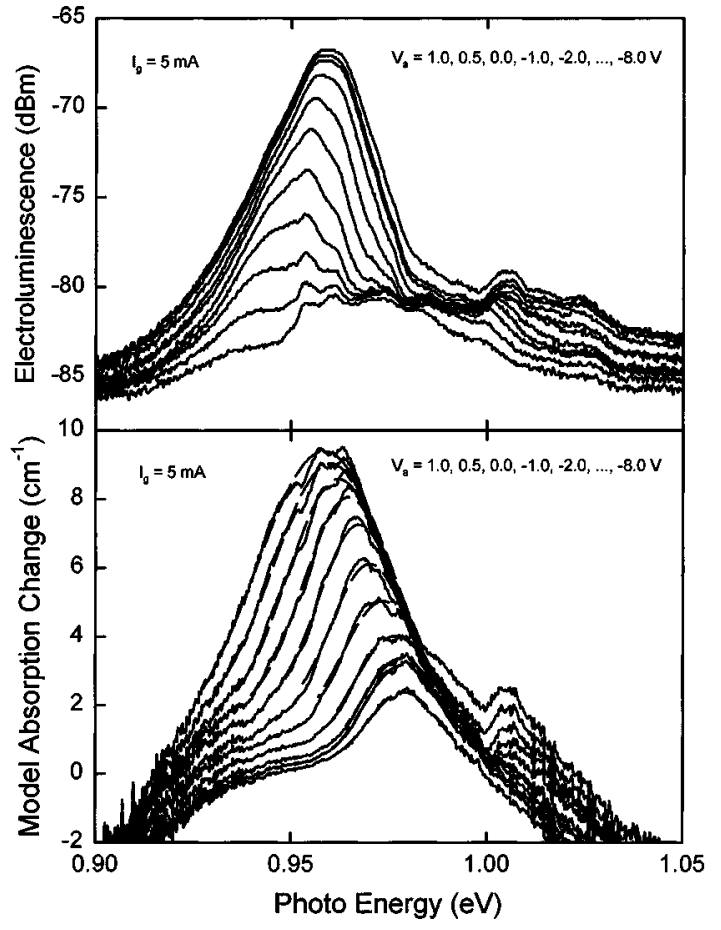

Fig. 4. (a) Electroluminescence from the absorber under constant laser injection current, $I_{g}=5 \mathrm{~mA}$ and with different reverse bias voltages applied to the absorber, $V_{a}=1.0,0.5,0.0,-1.0,-2.0, \ldots,-8.0 \mathrm{~V}$. (b) Calculated modal absorption spectra, or change in modal absorption loss $\Delta \alpha$, of the absorber under different reverse bias conditions.

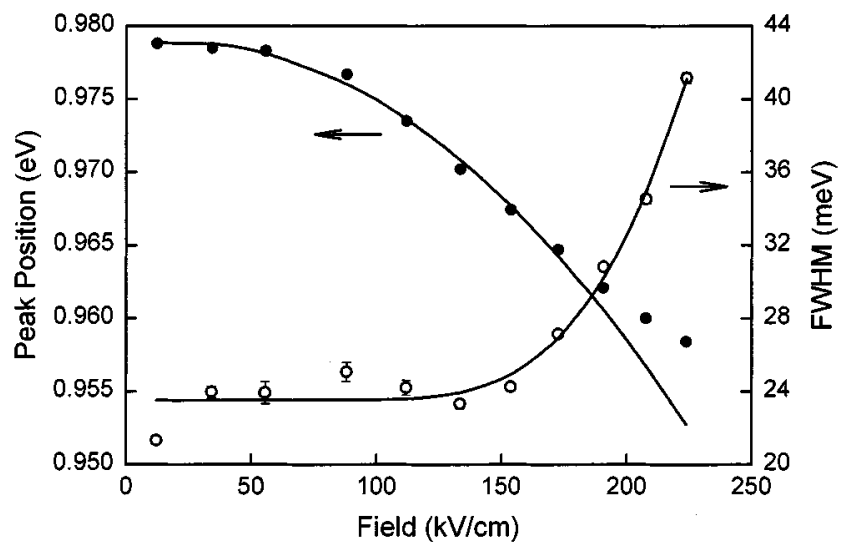

Fig. 5. Absorption spectrum peak position (in dark circles) and FWHM linewidth (in open circles) as a function of the internal electrical field. Error bars indicate the precision to which the data points could be determined from Gaussian envelope fitting. Solid lines are fit curves where a quadratic dependence and an exponential relation were assumed for the peak position and linewidth broadening, respectively.

$\left(I_{g}=5 \mathrm{~mA}\right)$. Fig. 4(a) shows the electroluminescence intensity from the absorber decreases as the bias voltage of the absorber changes from forward bias, $V_{a}=1.0 \mathrm{~V}$, to reverse bias, $V_{a}=-8.0 \mathrm{~V}$. In the mean time, the electroluminescence peak position red-shifts to a smaller photon energy. The modal absorption spectra, or strictly, the changes in modal absorption loss, $\Delta \alpha$, under different reverse bias conditions were calculated using the following equation:

$$
\Delta \alpha=-\frac{1}{L_{a}} \ln \left(\frac{I}{I_{\text {ref }}}\right)
$$

in which the cavity resonance was ignored, and the results are shown in Fig. 4(b) Here, $I$ is the electroluminescence intensity at a certain bias voltage, and $I_{\text {ref }}$ is the reference intensity level corresponding to the electroluminescence of the gain section under transparency, where any absorption or gain present is small. The peak position of $\Delta \alpha$ is actually the ground state transition peak of the absorption spectra. Fig. 4(b) shows both the redshift of the spectral absorption peak and the broadening of the absorption spectrum with increasing reverse bias. The absorption spectra peaks were fitted with Gaussian envelopes, shown as dashed lines in Fig. 4(b). For a higher reverse bias voltage, e.g., $\left|V_{a}\right| \geq 4.0 \mathrm{~V}$, a smaller peak arises at higher photon energy, corresponding to the absorption due to an excited state transition.

The redshift and broadening of the absorption spectrum under reverse bias conditions can be attributed to the QCSE, arising from the electric field in the QD layers. The internal electrical field across the QD was calculated for the laser structure under different reverse bias conditions. The ground state absorption peak position and the full-width at half-maximum (FWHM) of the absorption spectra were extracted from the Gaussian fitting parameters and plotted against the internal electrical field in Fig. 5. The peak positions at a reverse bias of $\left|V_{a}\right| \leq 5 \mathrm{~V}$ can be approximately described by a quadratic dependence. The peak redshift deviates from the quadratic dependence at higher reverse biases, possibly because the field is large enough to begin dissociating the electron-hole pair and thus influences the transition energy [16]. The Stark shift has an amplitude of $\sim 10 \mathrm{meV}$ under an electric field of $150 \mathrm{kV} / \mathrm{cm}$, which is comparable with the measurement of an InAs QD on GaAs in [17], and smaller than that in an ordinary QW under the same electric field [18]. This can be explained by the relatively high barrier height and the small size of the QD's along the electrical field [16]. The FWHM of the absorption spectra change only slightly at low electrical fields (less than $150 \mathrm{kV} / \mathrm{cm}$ ), and rise sharply at higher fields.

This linewidth increase is attributed to a reduced lifetime of the exciton in the dot caused by the tunneling of carriers out of the dot [19]. The expression below, derived in [19] for linewidth broadening of a single QD under an electrical field, was used to fit the linewidth broadening of the absorption spectra peaks

$$
\Gamma=\Gamma_{0}+a \exp \left(-\frac{b}{F}\right) .
$$

Here, $\Gamma$ is the linewidth as a function of electrical field, $F, \Gamma_{0}=23.52 \mathrm{meV}$ is the linewidth at zero field, and $a=4517.2 \mathrm{meV}$ and $b=1242.03 \mathrm{kV} / \mathrm{cm}$ are fitting parameters. The fit indicates that the broadening of the absorption spectra follows the same dependence as linewidth broadening of a single QD. 


\section{CONCLUSION}

In summary, long wavelength, oxide-confined two-section QD lasers with an integrated intracavity QD saturable absorber have been fabricated. In addition to achieving efficient room temperature $\mathrm{CW}$ lasing operation in the ground state, hysteresis and bistability have also been observed in both the $L-I_{g}$ characteristics of the laser voltage, and in the reverse-biased characteristics of the light-absorber. The origin of bistable operation in the laser's $L-I_{g}$ characteristic was attributed to the nonlinear saturation of the QD absorption, presumably caused by state filling. The bistability of the light-absorber's reverse-biased characteristics is due to both nonlinear absorption saturation and the electroaborption induced by the QCSE under the applied electrical field. The QD absorption spectra have been measured and the QCSE has been observed. The redshift and broadening of the absorption spectra have been obtained from the Gaussian envelope fitting of the absorption spectra as a function of internal electrical field. The Stark shift of the absorption spectra follows a quadratic dependence at small electric fields, while the linewidth broadening of the absorption spectra has the same electric field dependence as the linewidth broadening of a single QD.

\section{REFERENCES}

[1] Yu. M. Shernyakov, D. A. Bedarev, E. Yu. Kondrat'eva, P. S. Kop'ev, A. R. Kovsh, N. A. Maleev, M. V. Maximov, S. S. Mikhrin, A. F. Tsatsul'nikov, V. M. Ustinov, B. V. Volovik, A. E. Zhukov, Zh. I. Alferov, N. N. Ledentsov, and D. Bimberg, "1.3 $\mu \mathrm{m}$ GaAs-based laser using quantum dots obtained by activated spinodal decomposition," Electron. Lett., vol. 35, pp. 898-900, 1999.

[2] L. F. Lester, A. Stintz, H. Li, T. C. Newell, E. A. Pease, B. A. Fuchs, and K. J. Malloy, "Optical characteristics of $1.24 \mu \mathrm{m}$ quantum dot lasers," IEEE Photon. Technol. Lett., vol. 11, pp. 931-933, 1999.

[3] K. Mukai, Y. Nakata, K. Otsubo, M. Sugawara, N. Yokoyama, and H. Ishikawa, "1.3- $\mu \mathrm{m} \mathrm{CW}$ lasing of InGaAs-GaAs quantum dots at room temperature with a threshold current of $8 \mathrm{~mA}$," IEEE Photon. Technol. Lett., vol. 11, pp. 1205-1207, Oct. 1999.

[4] G. Park, O. B. Shchekin, D. L. Huffaker, and D. G. Deppe, "Low-threshold oxide-confined $1.3-\mu \mathrm{m}$ quantum dot laser," IEEE Photon. Technol. Lett., vol. 13, pp. 230-232, Mar. 2000.

[5] X. Huang, A. Stintz, C. P. Hains, G. T. Liu, J. Cheng, and K. J. Malloy, "Efficient high temperature $\mathrm{CW}$ lasing operation of oxide-confined long-wavelength InAs quantum dot lasers," Electron. Lett., vol. 36, no. 1, pp. 41-42, 2000.

[6] O. Qasaimeh, W. D. Zhou, J. Philips, S. Krishna, P. Bhattacharya, and M. Dutta, "Bistability and self-pulsation in quantum-dot lasers with intracavity quantum-dot saturable absorbers," Appl. Phys. Lett., vol. 74, no. 12 , pp. $1654-1656,1999$.

[7] H. Kawaguchi, "Bistable laser diodes and their application: State of the art," IEEE J. Select. Topics Quantum Electron., vol. 3, pp. 1254-1270, 1997.

[8] — "Progress in optical functional devices using two-section laser diodes/amplifiers," in IEE Proc. J, vol. 140, 1993, pp. 3-15.

[9] L. Zhang, T. F. Boggess, D. G. Deppe, D. L. Huffaker, O. B. shchekin, and C. Cao, "Dynamic response of 1.3- $\mu$ m-wavelength InGaAs/GaAs quantum dots," Appl. Phys. Lett., vol. 76, no. 10, pp. 1222-1224, 2000.

[10] J. M. Dallesasse, N. Holonyak, A. R. Sugg, and T. A. Richard, "Hydrolyzation oxidation of AlGaAs-AlAs-GaAs quantum well heterostructures and superlattices," Appl. Phys. Lett., vol. 57, no. 26, pp. 2844-2846, 1990
[11] A. Schmitt-Rink, D. A. B. Miller, and D. S. Chemla, "Theory of the linear and nonlinear optical properties of semiconductor microcrystallites," Phys. Rev. B, vol. 35, no. 15, pp. 8113-8125, 1987.

[12] T. Matsumoto, M. Ohtsu, K. Matsuda, T. Saiki, H. Saito, and K. Nishi, "Low-temperature near-field nonlinear absorption spectroscopy of InGaAs single quantum dots," Appl. Phys. Lett., vol. 75, no. 21, pp. 3246-3248, 1999

[13] H. Uenohara, R. Takahashi, Y. kawamura, and H. Iwamura, "Static and dynamic response of multiple-quantum-well voltage controlled bistable laser diodes," IEEE J. Quantum Electron., vol. 32, pp. 873-883, May 1996.

[14] E. Goutain, J. C. Renaud, M. Krakowski, D. Rondi, R. Blondeau, and D. Decoster, "30 GHz bandwidth, $1.55 \mu \mathrm{m}$ MQW-DFB laser diode based on a new modulated scheme," Electron. Lett., vol. 32, no. 10, pp. 896-897, 1996.

[15] S. D. McDougall and C. N. Ironside, "Measurements of reverse and forward bias absorption and gain spectra in semiconductor laser material," Electron. Lett., vol. 31, no. 25, pp. 2179-2181, 1995.

[16] G. W. Wen, J. Y. Lin, H. X. Jiang, and Z. Zhen, "Quantum-confined Stark effects in semiconductor quantum dots," Phys. Rev. B, vol. 52, no. 8, pp. 5913-5922, 1995.

[17] P. W. Fry, I. E. Itskevich, D. J. Mowbray, M. S. Skolnick, J. J. Finley, J. A. Barker, E. P. O'Reilly, L. R. Wilson, I. A. Larkin, P. A. Maksym, M. Hopkinson, M. Al-Khafaji, J. P. R. David, A. G. Cullis, G. Hill, and J. C. Clark, "Inverted electron-hole alignment in InAs-GaAs Self-assembled quantum dats," Phys. Rev. Lett., vol. 84, no. 4, pp. 733-736, 2000.

[18] R. Y.-F. Yip, P. Desjardins, L. Isnard, A. Ait-Ouali, H. Marchand, J. L. Brebner, J. F. Currie, and R. A. Masut, "Band alignment engineering for high speed, low drive field quantum confined Stark effect devices," $J$. Appl. Phys., vol. 83, no. 3, pp. 1758-1769, 1998.

[19] W. Heller, U. Bockelmann, and G. Abstreiter, "Electric-field effects on excitons in quantum dots," Phys. Rev. B, vol. 57, no. 11, pp. 6270-6273, 1998.

Xiaodong Huang, photograph and biography not available at the time of publication.

A. Stintz, photograph and biography not available at the time of publication.

Hua $\mathbf{L i}$, photograph and biography not available at the time of publication.

Audra Rice, photograph and biography not available at the time of publication

G. T. Liu, photograph and biography not available at the time of publication.

L. F. Lester, photograph and biography not available at the time of publication.

Julian Cheng, photograph and biography not available at the time of publication.

K. J. Malloy, photograph and biography not available at the time of publication. 